\title{
Terahertz Local Tomography
}

\author{
Xiaoxia Yin, Brian W.-H. Ng, Bradley Ferguson, and Derek Abbott
}

\begin{abstract}
We use the theory of two dimensional discrete wavelet transforms to derive inversion formulas for the Radon transform of terahertz datasets. These inversion formulas with good localised properties are implemented for the reconstruction of terahertz imaging in the area of interest, with a significant reduction in the required measurements. As a form of optical coherent tomography, terahertz CT complements the current imaging techniques and offers a promising approach for achieving non-invasive inspection of solid materials, with potentially numerous applications in industrial manufacturing and biomedical engineering [7], [2], [6].
\end{abstract}

Index Terms - Terahertz, T-rays, wavelet, computed tomography (CT), filtered back projection (FBP)

\section{INTRODUCTION}

This paper for CT reconstruction is motivated by terahertz time domain spectroscopy (TDS) imaging mechanisms and focuses on terahertz CT imaging with reduced projection angles. The main goal of this paper is to present a wavelet based reconstruction algorithm for terahertz computed tomography and to show how this algorithm can be used to rapidly reconstruct the region of interest (ROI) with a reduction in the measurements of terahertz responses, compared with a standard reconstruction. The current algorithm provides new insight into the relationship between local reconstruction, local projection, and the resolution of terahertz coherent tomography. This algorithm is sensitive to terahertz data when reconstructing local projections using wavelet techniques, resulting in variations in the boundary of the local projection region after the wavelet transform, which gives rise to different resolution and reconstructed image sizes.

\section{IMPLEMENTATION}

\section{A. Practical Consideration}

In local reconstruction [3], [5], artifacts are common close to the boundary of the ROE, after applying a filter on truncated data. The situation is more serious especially for imaging local terahertz CT measurement. In order to validate local reconstruction of terahertz measurement using our algorithm, the target sample of a polystyrene cylinder with holes drilled is investigated. The terahertz measurement of the current sample consists of 101 projections at each of 25 projection angles cover $180^{\circ}$ projection area. The resultant reconstruction has the image size of $100 \times 100$ pixels with the pixel interval at $0.5 \mathrm{~mm}$.

Centre for Biomedical Engineering and School of Electrical \& Electronic Engineering, The University of Adelaide, SA 5005, Australia e-mail: (dabbott@eleceng.adelaide.edu.au).
The current wavelet [4], [1] based reconstruction algorithm is to assume that support of the image is a disc of radius $R$, and the radius of the ROI is $r_{i}$. The radius $r_{e}=r_{i}+r_{a}$ is exposed, where $r_{a}$ is the extra margin with related to the radius of $\mathrm{ROA}$, produced due to applying wavelet filters on projection data at a low resolution. The region of exposure (ROE) is calculated via setting the outside of ROE to zero. A wavelet ramp filtered back projection formula is applied to the remaining projections, for wavelet based reconstruction.

The algorithm is summarized as follows.

1) The original projects are calculated based on the measured desired parameters of terahertz imaging.

2) The region of exposure is truncated for the reconstruction of an image in the region of interest.

3) the region of exposure of each projection is filtered by modified scaling and wavelet filters at all projection angles. This step is to recover an image related to approximate and detailed reconstructed coefficients.

4) The projections at the step 4 are linearly extrapolated to delete artifiacts along the borders of the ROE in the projections.

5) Filtered projections are obtained in step 3 and step 4 are back projected to every other point, using wavelet ramp filter, to obtain the approximate and detail coefficients at resolution $2^{-1}$. The remaining points are set to zero.

6) The image is reconstructed from the wavelet and scaling coefficients by normal inverse 2D wavelet transforms.

1) Exploration of the Processing of Terahertz Signogram: The difficulty for the local reconstruction using terahertz techniques is that there exists sharp variation along the borders of the ROE after applying wavelet ramp filters, on each of the 1D projections, which result in invisible reconstructed images at the ROI. To overcome this problem, To overcome this problem, an extrapolation of the projections is performed. The ROA is a range of radius $r_{a}$ for convenience. Let us assume that the ROE is the subset of projections on which $s_{\theta}$ is given and it is a circle of radius $r_{e}$ whose center is located at polar coordinates $\left(r, \theta_{0}\right)$. It holds that

$$
\begin{array}{r}
(R O E-R O A):\left\{p: p \in\left[r \cos \left(\theta-\theta_{0}\right)-\left(r_{e}-r_{a}\right),\right.\right. \\
\left.\left.r \cos \left(\theta-\theta_{0}\right)+\left(r_{e}-r_{a}\right)\right]\right\} .
\end{array}
$$

The constant extrapolation satisfies 


$$
\left(s_{\theta}\right)_{\text {local }}(p)=\left\{\begin{array}{l}
s_{\theta}(p) \\
\text { if } p \in(\mathrm{ROE}-\mathrm{ROA}), \\
s_{\theta}\left(r \cos \left(\theta-\theta_{0}\right)+\left(r_{e}-r_{a}\right)\right) \\
\text { if } p \in\left[r \cos \left(\theta-\theta_{0}\right)+\left(r_{e}-r_{a}\right)\right], \\
s_{\theta}\left(r \cos \left(\theta-\theta_{0}\right)-\left(r_{e}-r_{a}\right)\right) \\
\text { if } p \in\left[r \cos \left(\theta-\theta_{0}\right)-\left(r_{e}-r_{a}\right)\right] .
\end{array}\right.
$$

The extrapolation algorithm fits both reconstruction of both centered areas and off-centered areas. A reconstruction of the centered area using our algorithm is illustrated for this paper.

\section{B. Local Reconstruction at Centered Area}

A centered disk radius of 16 pixels of the polystyrene phantom is recovered from the extrapolated scaling and wavelet coefficients using local reconstruction method proposed in this paper, with $46 \%$ of full projection data. The wavelet based reconstructed image with size of $196 \times 196$ pixels is shown in Fig. 1(b) after extrapolation. Extrapolated wavelet and scaling coefficients after back projection are shown in Fig. 1(c), each of which is evaluated on a $100 \times 100$ image. For comparison, Fig. 1(a) illustrates the global reconstruction of a $196 \times 196$ pixel image, using traditional FBP algorithm. It is visually comparable that our algorithm allows as satisfactory local reconstruction as traditional FBP algorithm and approximate reconstruction from scaling function shows good local features for the reconstruction in the region of interest.

\section{Conclusion}

We have developed an algorithm to reconstruct the wavelet and scaling coefficients of a function from its Radon transform of terahertz measurement. Based on the observation that for some wavelet bases with sufficiently many zero moments, the scaling and wavelet functions have essentially the same support after ramp filtering. The local reconstruction scheme is effective to recover a cross section of a target object at a local region with essentially local data of terahertz measurement. Scaling function shows well localised features compared to the traditional FBP for local reconstruction. Wavelet reconstruction of an image from scaling and wavelet filters enable a satisfactory image.

\section{REFERENCES}

[1] D. L. Donoho. De-noising by soft thresholding. IEEE Transactions on Information Theory, 41(3):613-627, 1995.

[2] B. Ferguson, S. Wang, D. Gray, D. Abbott, and X. C. Zhang. Identification of biological tissue using chirped probe $\mathrm{THz}$ imaging. Microelectronics Journal (Elsevier), 33(12):1043-1051, 2002.

[3] M. Holschneider. Inverse radon transforms through inverse wavelet transforms. Inverse Problems, 7:853-861, 1991.

[4] S. Mallat. A Wavelet Tour of Signal Processing. Academic Press, San Diego, CA., 1999.

[5] F. Rashid-Farrokhi, K.J.R. Liu, C.A. Berenstein, and D. Walnut. Wavelet-based multiresolution local tomography. IEEE Transactions on Image Processing, 6(10):1412-1430, 1997.

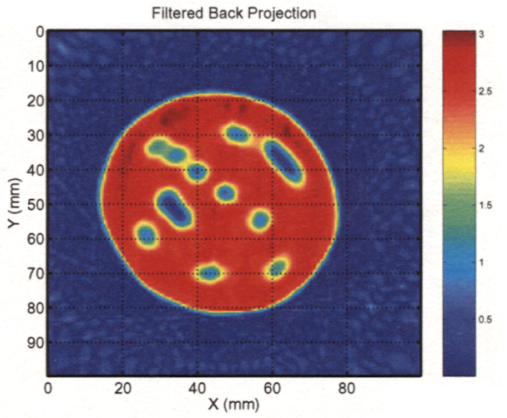

(a)

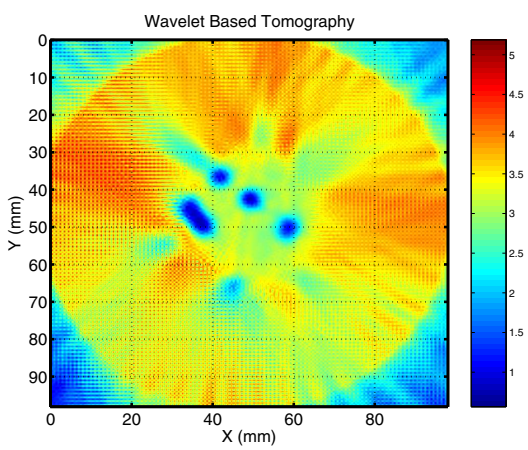

(b)
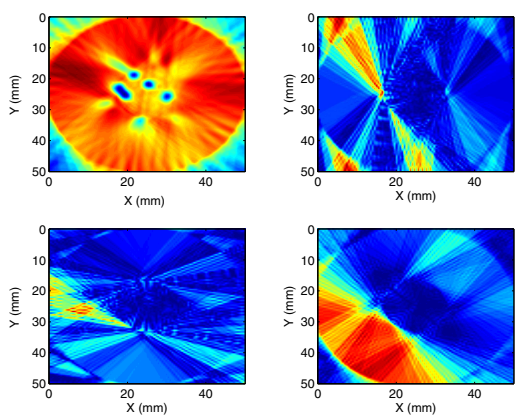

(c)

Fig. 1. (a)A global reconstruction using a traditional FBP algorithm. (b)A wavelet based reconstruction. (b)Reconstruction images from local scaling and wavelet coefficients after back projection.

[6] H.P. Siegel. Terahertz technology in biology and medicine. IEEE transactions on microwave theory and techniques, 52(10):24382447, 2004.

[7] B. Withayachumnankul, B. Ferguson, T. Rainsford, D. Findlay, S. P. Mickan, and D. Abbott. T-ray relevant frequencies for osteosarcoma classification. In Process of SPIE Photonics: Design, Technology, and Packaging II, volume 6038, page art. no. 60381H, Adelaide, Australia, 2005. 